\title{
DUO, BÉZOUT AND DISTRIBUTIVE RINGS OF SKEW POWER SERIES
}

\author{
Ryszard Mazurek and Michą Ziembowski
}

\begin{abstract}
We give necessary and sufficient conditions on a ring $R$ and an endomorphism $\sigma$ of $R$ for the skew power series ring $R[[x ; \sigma]]$ to be right duo right Bézout. In particular, we prove that $R[[x ; \sigma]]$ is right duo right Bézout if and only if $R[[x ; \sigma]]$ is reduced right distributive if and only if $R[[x ; \sigma]]$ is right duo of weak dimension less than or equal to 1 if and only if $R$ is $\aleph_{0}$-injective strongly regular and $\sigma$ is bijective and idempotent-stabilizing, extending to skew power series rings the Brewer-Rutter-Watkins characterization of commutative Bézout power series rings.
\end{abstract}

\section{Introduction and the main result}

The aim of this paper is to characterize skew power series rings that are right duo right Bézout, which by the main result of the paper, Theorem 1.6, is the same as to characterize skew power series rings that are right duo right distributive as well as to characterize right duo skew power series rings of weak dimension less than or equal to 1 .

In this introductory section we recall those results on (skew) power series rings that motivated our studies and which we generalize in this paper. Here we also formulate our main result, Theorem 1.6, in which we give some equivalent conditions for a skew power series ring to be right duo right Bézout. This characterization theorem is proved in Section 3. In Section 2 we collect results on skew power series rings that are right Bézout, or right duo, or reduced, or whose coefficient ring is $\aleph_{0}$-injective strongly regular, which we apply in the proof of the main result.

All rings in this paper are associative with unity. Recall that a ring $R$ is right (left) Bézout if all its finitely generated right (left) ideals are

2000 Mathematics Subject Classification. Primary: 16W60; Secondary: 16D50, $16 \mathrm{E} 50$.

Key words. Skew power series ring, right Bézout ring, right distributive ring, right duo ring. 
principal, and $R$ is a Bézout ring if it is right and left Bézout. A ring $R$ is right (left) $\aleph_{0}$-injective provided any homomorphism from a countably generated right (left) ideal of $R$ into $R$ extends to a right (left) $R$-module endomorphism of $R$. By an $\aleph_{0}$-injective ring we mean a right and left $\aleph_{0}$-injective ring.

In [1] J. W. Brewer, E. A. Rutter and J. J. Watkins proved that for any commutative power series ring $R[[x]]$ the following equivalences hold:

$$
R[[x]] \text { is Bézout }
$$

文

$R$ is $\aleph_{0}$-injective von Neumann regular

业

$R[[x]]$ has weak dimension less than or equal to 1 ,

where a ring $R$ is said to be von Neumann regular if $a \in a R a$ for any $a \in R$.

Bézout power series rings in the noncommutative setting were studied by D. Herbera in [3], where among other results she proved a generalization of the Brewer, Rutter and Watkins result, which we quote below (see also [10] for more general results).

Recall that a ring $R$ is right (left) duo if all right (left) ideals of $R$ are two-sided ideals, and $R$ is duo if it is right and left duo. Recall also that a ring $R$ is abelian if all idempotents of $R$ are central, and a ring $R$ is strongly regular if $a \in a^{2} R$ for any $a \in R$. Strongly regular rings are precisely abelian von Neumann regular rings. Since every element of a strongly regular ring $R$ is a product of a unit and a central idempotent, it follows that strongly regular rings are duo, and for strongly regular rings the $\aleph_{0}$-injectivity is a right-left symmetric condition (see Subsection 2.3).

Theorem 1.1 (Herbera; see [3, Corollary 1.10]). Let $R$ be a strongly regular ring. The following conditions are equivalent:

(1) $R[[x]]$ is right Bézout.

(2) $R[[x]]$ is Bézout.

(3) $R[[x]]$ has weak dimension less than or equal to 1.

(4) $R$ is $\aleph_{0}$-injective.

In this situation $R[[x]]$ is duo and all ideals of $R[[x]]$ are generated by central elements.

Duo Bézout power series rings appeared also in a characterization of $\aleph_{0}$-injective strongly regular rings given by O. A. S. Karamzadeh and A. A. Koochakpoor in [5], where they proved the following theorem. 
Theorem 1.2 (Karamzadeh and Koochakpoor; see [5, Theorem 1.10]). Let $R$ be a strongly regular ring. Then $R$ is $\aleph_{0}$-injective if and only if $R[[x]]$ is duo and Bézout.

In [9] G. Marks studied the duo property for Ore extensions of rings, and among other results he proved the following theorem.

Theorem 1.3 (Marks; see [9, Proposition 5]). Let $R$ be a left or right self-injective von Neumann regular ring. The following conditions are equivalent:

(1) $R[[x]]$ is right duo.

(2) $R[[x]]$ is left duo.

(3) $R$ is right duo.

(4) $R$ is left duo.

In [13] and [14] A. A. Tuganbaev extended the previously mentioned result of Brewer, Rutter and Watkins to skew power series rings, adding additionally the right distributivity property to the list of equivalences, and showing that the condition concerning the weak dimension can be replaced with a weaker property of 2-generated right ideals (i.e., right ideals generated by two elements). To quote those results of Tuganbaev, we need some definitions.

Let $R$ be a ring. Then $R$ is said to be right (left) distributive if the lattice of right (left) ideals of $R$ is distributive, i.e., $(I+J) \cap K=$ $(I \cap K)+(J \cap K)$ holds for any right (left) ideals $I, J, K$ of $R$. We say that $R$ is distributive if $R$ is right and left distributive. The ring $R$ is called semicommutative if the right annihilator of every element of $R$ is a two-sided ideal (clearly the notion is left-right symmetric). Recall that $R$ is said to be right (left) quasi-duo if every maximal right (left) ideal of $R$ is a two-sided ideal. For an endomorphism $\sigma$ of the ring $R$ the skew power series ring over $R$ in one variable $x$ is denoted by $R[[x ; \sigma]]$. Thus elements of $R[[x ; \sigma]]$ are power series of the form $\sum_{n=0}^{\infty} a_{n} x^{n}$, where all the coefficients $a_{n}$ belong to $R$, with multiplication defined by the rule $x a=\sigma(a) x$ for any $a \in R$.

Theorem 1.4 (Tuganbaev; see [14, Theorem 2]). Let $\sigma$ be an injective endomorphism of a ring $R$. Then the following conditions are equivalent:

(1) $R[[x ; \sigma]]$ is right Bézout and $R$ is semicommutative.

(2) $R[[x ; \sigma]]$ is right Bézout and $R$ is right quasi-duo.

(3) $R[[x ; \sigma]]$ is right distributive.

(4) $R$ is $\aleph_{0}$-injective strongly regular, $\sigma$ is bijective and $\sigma(e)=e$ for any idempotent $e=e^{2} \in R$. 
Theorem 1.5 (Tuganbaev; see [13, Theorem 1]). Let $R$ be an abelian ring and $\sigma$ an automorphism of $R$ such that $\sigma(e)=e$ for any idempotent $e=e^{2} \in R$. Then the following conditions are equivalent:

(1) $R[[x ; \sigma]]$ has weak dimension less than or equal to 1.

(2) All 2-generated right ideals of $R[[x ; \sigma]]$ are flat.

(3) $R$ is $\aleph_{0}$-injective strongly regular.

In this paper we show that in a sense all the conditions appearing in the results quoted above (starting from this of Brewer, Rutter and Watkins) are equivalent. More precisely, these results are consequences of the following theorem, which being the main result of the paper, specifies eleven equivalent conditions for a skew power series ring $R[[x ; \sigma]]$ to be right duo right Bézout. We would like to emphasize that besides conditions suggested by the quoted results, the theorem also contains some new ones. We would also like to stress that according to the second part of this theorem, each of the equivalent conditions implies its left analogue (but need not be equivalent to it; see Example 3.3), and if $\sigma$ is an automorphism, then any condition in this theorem is equivalent to its left analogue. To prove this theorem we need some results on special classes of skew power series rings which we assemble in Section 2, and we apply them in Section 3 in the proof of the theorem.

Recall that a ring $R$ is reduced if it contains no nonzero nilpotent element, i.e., $a^{2}=0$ implies $a=0$ for any $a \in R$.

Theorem 1.6. Let $\sigma$ be an endomorphism of a ring $R$. Then the following conditions are equivalent:

(1) $R[[x ; \sigma]]$ is right Bézout and right duo.

(2) $R[[x ; \sigma]]$ is right Bézout and reduced.

(3) $R[[x ; \sigma]]$ is right Bézout and right quasi-duo, and $\sigma$ is injective.

(4) $R[[x ; \sigma]]$ is right Bézout and semicommutative, and $\sigma$ is injective.

(5) $R[[x ; \sigma]]$ is right Bézout and abelian, and $\sigma$ is injective.

(6) $R[[x ; \sigma]]$ is right distributive and right duo.

(7) $R[[x ; \sigma]]$ is right distributive and reduced.

(8) $R[[x ; \sigma]]$ is right distributive and $\sigma$ is injective.

(9) All 2-generated right ideals of $R[[x ; \sigma]]$ are flat, $R$ is abelian, $\sigma$ is bijective and $\sigma(e)=e$ for any $e=e^{2} \in R$.

(10) $R[[x ; \sigma]]$ has weak dimension less than or equal to 1 and $R[[x ; \sigma]]$ is right duo. 
(11) $R[[x ; \sigma]]$ has weak dimension less than or equal to $1, R$ is abelian, $\sigma$ is bijective and $\sigma(e)=e$ for any $e=e^{2} \in R$.

(12) $R$ is $\aleph_{0}$-injective strongly regular, $\sigma$ is bijective and $\sigma(e)=e$ for any $e=e^{2} \in R$.

Furthermore, these conditions imply the left analogues of (1)-(10), and in particular, if any of these conditions holds, then $R[[x ; \sigma]]$ is duo. Moreover, if $\sigma$ is bijective, then these conditions are equivalent to the left analogues of (1)-(10).

\section{Special classes of skew power series rings}

In this section we prove some results on special classes of skew power series rings that will be used in the proof of Theorem 1.6 in the next section.

2.1. Right Bézout rings of skew power series. In Section 3 we will need the following generalization of Herbera's result [3, Lemma 2.2]. Recall that a ring $R$ is Dedekind-finite if for any $a, b \in R, a b=1 \mathrm{im}$ plies $b a=1$.

Proposition 2.1. Let $R$ be a Dedekind-finite ring and $\sigma$ an injective endomorphism of $R$ such that $R[[x ; \sigma]]$ is right Bézout. Then $R$ is von Neumann regular.

Proof: Set $A=R[[x ; \sigma]]$. For any $f=\sum_{n=0}^{\infty} f_{n} x^{n} \in A$ we set $\hat{f}=$ $\sum_{n=0}^{\infty} \sigma\left(f_{n}\right) x^{n}$, i.e., $\hat{f}$ is a unique element of $A$ such that $x f=\hat{f} x$. It is clear that $\widehat{f g}=\hat{f} \hat{g}$ for any $f, g \in A$.

We claim that (cf. [3, Proposition 2.1])

for any $f=\sum_{n=0}^{\infty} f_{n} x^{n}, g=\sum_{n=0}^{\infty} g_{n} x^{n} \in A$, if $f g=x$, then $\hat{g} f=x$.

To see this, note that since $A$ is right Bézout, the right ideal $g A+x A$ is principal and thus there exist $h=\sum_{n=0}^{\infty} h_{n} x^{n}, k=\sum_{n=0}^{\infty} k_{n} x^{n}, \alpha=$ $\sum_{n=0}^{\infty} \alpha_{n} x^{n}, \beta \in A$ such that

$$
g=(g h+x k) \alpha \text { and } x=(g h+x k) \beta
$$


Multiplying the first part of (2.1) on the left by $f$, we obtain $x=(x h+$ $f x k) \alpha$, and equating $x$-coefficients, we see that

$$
1=\left[\sigma\left(h_{0}\right)+f_{0} \sigma\left(k_{0}\right)\right] \sigma\left(\alpha_{0}\right) .
$$

Since $R$ is Dedekind-finite, it follows that $\sigma\left(\alpha_{0}\right)$ is a unit of $R$, and thus $\hat{\alpha}$ is a unit of $A$ (see [7, p. 10]). Since (2.1) implies that

$$
\hat{g}=(\widehat{g h+x} k) \hat{\alpha} \text { and } x=(\widehat{h+x+x} k) \hat{\beta}
$$

we deduce that

$$
x=\hat{g} \gamma \text { for } \gamma=\hat{\alpha}^{-1} \hat{\beta}
$$

By assumption $f g=x$, and thus $\hat{f} \hat{g}=x$. Hence (2.3) implies that $\hat{f} x=x \gamma=\hat{\gamma} x$, and $\hat{f}=\hat{\gamma}$ follows. Since $\sigma$ is injective, we have $f=\gamma$, which proves our claim.

Now we are in a position to prove that $R$ is von Neumann regular. Let $a \in R$. Since $A$ is right Bézout, the right ideal $a A+x A$ is principal, and thus for some $p=\sum_{n=0}^{\infty} p_{n} x^{n}, q, \delta=\sum_{n=0}^{\infty} \delta_{n} x^{n}, \epsilon=\sum_{n=0}^{\infty} \epsilon_{n} x^{n} \in A$ we have

$$
a=(a p+x q) \delta \text { and } x=(a p+x q) \epsilon \text {. }
$$

Applying our claim to the second part of (2.4), we obtain $\hat{\epsilon}(a p+x q)=x$ and thus the first part of (2.4) implies the equality $\hat{\epsilon} a=x \delta$. Equating $x$-coefficients in the equality, we see that $\sigma\left(\epsilon_{1}\right) \sigma(a)=\sigma\left(\delta_{0}\right)$, and since $\sigma$ is injective, it follows that $\delta_{0}=\epsilon_{1} a$. On the other hand, equating constant terms in the first part of (2.4), we obtain $a=a p_{0} \delta_{0}$. Hence $a=a p_{0} \delta_{0}=a p_{0} \epsilon_{1} a \in a R a$.

2.2. Right duo rings of skew power series. Observe that for any ring we have the following implication relations:

$$
\text { right duo } \Rightarrow \text { semicommutative } \Rightarrow \text { abelian. }
$$

We will use this observation in the proof of the following proposition.

Proposition 2.2. Let $R$ be a ring and $\sigma$ an endomorphism of $R$. If $R[[x ; \sigma]]$ is right duo, then $R$ is right duo, $\sigma$ is bijective and $\sigma(e)=e$ for any $e=e^{2} \in R$.

Proof: Set $A=R[[x ; \sigma]]$. Since by assumption $A$ is right duo, for any $a, b \in R$ there exists $f=\sum_{n=0}^{\infty} f_{n} x^{n} \in A$ such that $b a=a f$. Hence $b a=a f_{0} \in a R$, proving that $R$ is right duo. 
To prove that $\sigma$ is bijective, we use an argument which is essentially due to Marks (see $[\mathbf{9}$, Proof of Theorem 1]). Since $A$ is right duo, for any $a \in R$ there exists $g \in A$ such that $a x=x g$. Equating $x$-coefficients in this equality, we obtain $a \in \sigma(R)$, and thus $\sigma$ is surjective. To show that $\sigma$ is injective, consider any $a \in R$ with $\sigma(a)=0$. Since $\sigma$ is surjective, $a=\sigma(b)$ for some $b \in R$. Since $A$ is right duo, in $R$ there exists a sequence $\left(c_{n}\right)_{n \in \mathbb{N}}$ such that

$$
a x+x^{3}=x\left(b+a x+x^{2}\right)=\left(b+a x+x^{2}\right)\left(c_{0}+c_{1} x+c_{2} x^{2}+c_{3} x^{3}+\cdots\right)
$$

Equating constant terms, $x$-, and $x^{3}$-coefficients in $(2.5)$, we obtain the following equations:

$$
0=b c_{0}, \quad a=b c_{1}+a \sigma\left(c_{0}\right), \quad 1=b c_{3}+a \sigma\left(c_{2}\right)+\sigma^{2}\left(c_{1}\right) .
$$

From the first part of (2.6) we obtain $0=\sigma\left(b c_{0}\right)=a \sigma\left(c_{0}\right)$, and thus the second part of (2.6) implies that $a=b c_{1}$, which leads to $0=a \sigma\left(c_{1}\right)$. Applying $\sigma^{2}$ to the third equation of (2.6), we obtain $\sigma^{4}\left(c_{1}\right)=1$. Hence, since $A$ is right duo, it follows that $x^{3}=x^{3} \sigma\left(c_{1}\right) \in \sigma\left(c_{1}\right) A$, and thus $1=\sigma\left(c_{1}\right) d$ for some $d \in R$. Hence $a=a\left(\sigma\left(c_{1}\right) d\right)=\left(a \sigma\left(c_{1}\right)\right) d=0$, proving that $\sigma$ is bijective.

Finally we show that if $e=e^{2} \in R$, then $\sigma(e)=e$. By the observation before Proposition 2.2, since $A$ is right duo, $e$ is central in $A$. Hence $e x=x e=\sigma(e) x$, proving that $e=\sigma(e)$.

2.3. Skew power series rings over $\aleph_{0}$-injective rings. In the proof of Theorem 1.6 we will need the following well known characterization of $\aleph_{0}$-injective strongly regular rings (see $[\mathbf{1 3}$, Lemmas 3,4$]$ ). Recall that a ring $R$ is said to be right $\aleph_{0}$-algebraically compact if for any system of a countable number of linear equations with a countable number of indeterminates and with coefficients from $R$ written on the left, if every finite subsystem of this system has a solution in $R$, then the whole system has a solution in $R$. This is to say that if $A$ is an $\aleph_{0} \times \aleph_{0}$ row-finite matrix over $R$, indexed by $\mathbb{N} \times \mathbb{N}, X$ is a column of $\aleph_{0}$ indeterminates and $B$ is a column of $\aleph_{0}$ elements of $R$, both indexed by $\mathbb{N}$, then the system $A X=B$ is finitely solvable if and only if it is solvable. Left $\aleph_{0}$-algebraically compact rings are defined analogously. It is well known that for a von Neumann regular ring $R$, the $\operatorname{ring} R$ is right (left) $\aleph_{0}$-algebraically compact if and only if it is left (right) $\aleph_{0}$-injective (see $[\mathbf{3}$, Proposition 1.1]). 
Proposition 2.3. For a strongly regular ring $R$, the following conditions are equivalent.

(1) $R$ is right $\aleph_{0}$-injective.

(2) $R$ is left $\aleph_{0}$-injective.

(3) $R$ is right $\aleph_{0}$-algebraically compact.

(4) $R$ is left $\aleph_{0}$-algebraically compact.

(5) For each sequence $\left(e_{n}\right)_{n=0}^{\infty}$ of orthogonal idempotents of $R$ and for each sequence $\left(a_{n}\right)_{n=0}^{\infty}$ of elements of $R$ there exists $b \in R$ such that be $e_{n}=a_{n} e_{n}$ for all $n \in \mathbb{N} \cup\{0\}$.

In the proof of Theorem 1.6 we will need also the following proposition.

Proposition 2.4. If $R$ is an $\aleph_{0}$-injective strongly regular ring and $\sigma$ is an automorphism of $R$ such that $\sigma(e)=e$ for every $e=e^{2} \in R$, then any principal right ideal of the ring $R[[x ; \sigma]]$ is generated by a power series whose coefficients are orthogonal idempotents of $R$.

Proof: Set $A=R[[x ; \sigma]]$ and let $f=\sum_{n=0}^{\infty} f_{n} x^{n} \in A$. Since $R$ is a strongly regular ring, for any $n \in \mathbb{N} \cup\{0\}$ there exist an idempotent $d_{n} \in$ $R$ and a unit $u_{n} \in R$ such that $f_{n}=d_{n} u_{n}$. Set

$$
e_{0}=d_{0} \text { and } e_{n}=d_{n}\left(1-d_{n-1}\right)\left(1-d_{n-2}\right) \cdots\left(1-d_{0}\right) \text { for } n \in \mathbb{N},
$$

and let

$$
g=\sum_{n=0}^{\infty} e_{n} x^{n} \in A .
$$

Since $e_{0}, e_{1}, e_{2}, \ldots$ are orthogonal idempotents, to complete the proof it suffices to show that $f A=g A$.

Since $R$ is $\aleph_{0}$-injective strongly regular, by Proposition 2.3 there exists a sequence $\left(b_{n}\right)_{n=0}^{\infty}$ of elements of $R$ such that $e_{m} b_{n}=e_{m} \sigma^{-m}\left(f_{m+n}\right)$ for any $m, n \in \mathbb{N} \cup\{0\}$, and since $\sigma(e)=e$ for any idempotent $e \in R$, we obtain

$$
e_{m} \sigma^{m}\left(b_{n}\right)=e_{m} f_{m+n} \text { for any } m, n \in \mathbb{N} \cup\{0\} .
$$

We claim that $f=g h$, where $h=\sum_{n=0}^{\infty} b_{n} x^{n} \in A$. For this, write $g h=\sum_{n=0}^{\infty} c_{n} x^{n}$. Then applying (2.7) and the equality $f_{n}=d_{n} f_{n}$, we obtain

$$
c_{n}=\sum_{i+j=n} e_{i} \sigma^{i}\left(b_{j}\right)=\sum_{i+j=n} e_{i} f_{i+j}=\left(\sum_{i=0}^{n} e_{i}\right) f_{n}=\left[\left(\sum_{i=0}^{n} e_{i}\right) d_{n}\right] f_{n} .
$$


It is easy to see that $\sum_{i=0}^{n-1} e_{i}+\prod_{i=0}^{n-1}\left(1-d_{i}\right)=1$ for any $n \in \mathbb{N}$, and thus

$$
\left(\sum_{i=0}^{n} e_{i}\right) d_{n}=\left[\sum_{i=0}^{n-1} e_{i}+\prod_{i=0}^{n-1}\left(1-d_{i}\right)\right] d_{n}=1 \cdot d_{n}=d_{n} .
$$

Hence $c_{n}=d_{n} f_{n}=f_{n}$, and thus $g h=f$, which proves that $f A \subseteq g A$.

To prove the opposite inclusion, we have to show that there exists $p=\sum_{n=0}^{\infty} p_{n} x^{n} \in A$ such that $g=f p$. Equating $x^{n}$-coefficients of $g$ and $f p$ for all $n \in \mathbb{N} \cup\{0\}$, we obtain a system of a countable number of linear equations

$$
e_{n}=\sum_{i+j=n} f_{i} \sigma^{i}\left(p_{j}\right) \quad(n=0,1,2, \ldots) .
$$

Since $\sigma$ is an automorphism and $\sigma(e)=e$ for any idempotent $e \in R$, the system (2.8) can be written in the equivalent form

$$
e_{n}=\sum_{i+j=n} \sigma^{-n}\left(f_{i}\right) \sigma^{-j}\left(p_{j}\right) \quad(n=0,1,2, \ldots) .
$$

Hence to prove the existence of $p \in A$ with $g=f p$, it suffices to show that the system

$$
e_{n}=\sum_{i+j=n} \sigma^{-n}\left(f_{i}\right) x_{j} \quad(n=0,1,2, \ldots)
$$

has a solution in $R$. Since $R$ is $\aleph_{0}$-injective strongly regular, Proposition 2.3 implies that the system (2.9) has a solution if and only if any its finite subsystem has a solution. Thus it suffices to show that for any $m \in \mathbb{N} \cup\{0\}$ there exist $y_{0}, y_{1}, \ldots, y_{m} \in R$ such that

$$
e_{n}=\sum_{i+j=n} \sigma^{-n}\left(f_{i}\right) y_{j} \text { for } n=0,1, \ldots, m .
$$

To show that the finite system (2.10) has a solution, note that for any $i \in \mathbb{N} \cup\{0\}$,

$$
f e_{i}=e_{i} x^{i}\left[\sigma^{-i}\left(u_{i}\right)+\sigma^{-i}\left(f_{i+1}\right) x+\sigma^{-i}\left(f_{i+2}\right) x^{2}+\cdots\right] .
$$

Since $\sigma^{-i}\left(u_{i}\right)$ is a unit of $R$, the power series in the brackets is a unit of $A$, and thus $e_{i} x^{i}=f k_{i}$ for some $k_{i} \in A$. Therefore, setting $q_{m}=$ $\sum_{i=0}^{m} k_{i} \in A$, we obtain $e_{0}+e_{1} x+e_{2} x^{2}+\cdots+e_{m} x^{m}=f q_{m}$. Now it is easy to see that if $q_{m}=\sum_{n=0}^{\infty} a_{n} x^{n}$ and $y_{j}=\sigma^{-j}\left(a_{j}\right)$ for any $j \in \mathbb{N} \cup\{0\}$, then $y_{0}, y_{1}, \ldots, y_{m}$ is a solution of (2.10). 
2.4. Reduced rings of skew power series. Recall that an endomorphism $\sigma$ of a ring $R$ is said to be rigid if $a \sigma(a) \neq 0$ for every nonzero $a \in R$. This notion proved to be very useful in characterizing reduced skew polynomial rings and skew power series rings in the work of J. Krempa in [6] and C. Y. Hong, N. K. Kim and T. K. Kwak in [4], to whom the first part of the following proposition is essentially due.

Proposition 2.5. Let $R$ be a ring and $\sigma$ an endomorphism of $R$. Then

(i) $R[[x ; \sigma]]$ is reduced if and only if $\sigma$ is rigid.

(ii) If $R[[x ; \sigma]]$ is reduced, then $\sigma$ is injective and $\sigma(e)=e$ for any $e=e^{2} \in R$

Proof: (i) If $R[[x ; \sigma]]$ is reduced, then for any nonzero $a \in R$ we have $a \sigma(a) x^{2}=(a x)^{2} \neq 0$, which proves that $\sigma$ is rigid. Conversely, if $\sigma$ is rigid, then it can be easily shown that $R$ is reduced (see [4, p. 218]), and thus $R[[x ; \sigma]]$ is reduced by [6, Corollary 3.5$]$.

(ii) Obviously, $\sigma$ is injective by (i). Let $e=e^{2} \in R$. Since the $\operatorname{ring} R[[x ; \sigma]]$ is reduced, it is abelian, and thus $e x=x e=\sigma(e) x$, proving that $e=\sigma(e)$.

\section{Proof of the main result}

We are now in a position to prove the main result of the paper as stated in Section 1. In the proof we will use the following well known implication relations that hold for any ring:

$$
\begin{gathered}
\text { right duo } \Rightarrow \text { right quasi-duo } \\
\Downarrow \\
\text { reduced } \Rightarrow \text { semicommutative } \Rightarrow \text { abelian }
\end{gathered}
$$

Proof of Theorem 1.6: From Theorem 1.4 we obtain $(8) \Leftrightarrow(12)$, and by Theorem 1.5 we have (11) $\Leftrightarrow(9) \Leftrightarrow(12)$. Thus the conditions (8), (9), (11) and (12) are equivalent.

We continue the proof by showing first that

$$
(12) \Rightarrow R[[x ; \sigma]] \text { is right duo. }
$$

To prove (3.1), it suffices to show that for the ring $A=R[[x ; \sigma]]$ and any $f \in A$ we have $A f \subseteq f A$. By Proposition 2.4 we can assume that $f=\sum_{n=0}^{\infty} e_{n} x^{n}$, where $\left(e_{n}\right)_{n=0}^{\infty}$ is a sequence of orthogonal idempotents of $R$, and we have to show that $g f \in f A$ for any $g=\sum_{n=0}^{\infty} g_{n} x^{n} \in A$. Since $R$ is $\aleph_{0}$-injective strongly regular, by Proposition 2.3 there exists 
a sequence $\left(t_{m}\right)_{m=0}^{\infty}$ of elements of $R$ such that for any $m, n \in \mathbb{N} \cup\{0\}$ we have $e_{n} t_{m}=e_{n} \sigma^{-n}\left(g_{m}\right)$, or equivalently, $e_{n} \sigma^{n}\left(t_{m}\right)=e_{n} g_{m}$. Set $h=\sum_{m=0}^{\infty} t_{m} x^{m} \in A$. Now it is easy to verify that $g f=f h$, which completes the proof of (3.1).

Since $(8) \Leftrightarrow(12)$, it follows from $(3.1)$ that $(8) \Rightarrow(6)$. Since $(6) \Rightarrow$ (8) is a direct consequence of Proposition 2.2, we obtain $(8) \Leftrightarrow(6)$.

Using again the equivalence $(8) \Leftrightarrow(12)$, we deduce from Proposition $2.5(\mathrm{i})$ that $(8) \Rightarrow(7)$. Since the opposite implication is a consequence of Proposition 2.5(ii), (8) $\Leftrightarrow(7)$ follows.

At this point we know that the conditions (6)-(9), (11) and (12) are equivalent. Now we show that they are equivalent to the conditions (1) through (5).

By Theorem 1.4, (8) implies that $R[[x ; \sigma]]$ is right Bézout, and since $(8) \Leftrightarrow(12)$, the implication $(8) \Rightarrow(1)$ follows from (3.1). Hence applying the implication chart $(*)$ and Proposition 2.2 , we obtain $(8) \Rightarrow(1) \Rightarrow$ (3). Furthermore, since all right quasi-duo right Bézout rings are right distributive (see $[\mathbf{1 2}, 2.35]$ ), we deduce that $(3) \Rightarrow(8)$. Moreover, since we already know that $(8) \Leftrightarrow(7)$ and $(8) \Rightarrow(1)$, it follows that $(8) \Rightarrow(2)$. Hence, applying $(*)$ and Proposition $2.5(\mathrm{ii})$, we obtain $(8) \Rightarrow(2) \Rightarrow(4)$ $\Rightarrow(5)$. Since obviously (5) implies that $R$ is abelian, and thus Dedekindfinite, it follows from Proposition 2.1 that $R$ is strongly regular. Hence $R$ is semicommutative, and from Theorem 1.4 we obtain $(5) \Rightarrow(8)$. Thus we have shown that the conditions (1)-(5) are equivalent to (8).

By the above, the conditions (1)-(9), (11) and (12) are equivalent. By $(*),(10)$ implies that $R[[x ; \sigma]]$ is abelian, and thus so is $R$. Hence using Proposition 2.2 , we obtain $(10) \Rightarrow(11)$. On the other hand, we know that $(11) \Leftrightarrow(12)$, and thus it follows from (3.1) that $(11) \Rightarrow(10)$, proving that $(11) \Leftrightarrow(10)$. Hence the conditions (1)-(12) are equivalent.

Now we prove that these conditions imply the left analogues of (1)-(10). Assume that one of the conditions (1)-(12) is satisfied. Since by the first part of the theorem these conditions are equivalent, (12) is satisfied, and thus $R$ is $\aleph_{0}$-injective strongly regular, $\sigma$ is bijective and $\sigma(e)=e$ for any $e=e^{2} \in R$. Hence $R^{\text {op }}$, the opposite ring of $R$, is $\aleph_{0}$-injective strongly regular, $\sigma^{-1}$ is an automorphism of $R^{\text {op }}$ and $\sigma^{-1}(e)=e$ for any $e=e^{2} \in R$. Thus (12) holds for the ring $R^{\text {op }}$ and its automorphism $\sigma^{-1}$. Hence, again by the first part of the theorem, any of the conditions (1)-(10) holds for the $\operatorname{ring} R^{\text {op }}$ and its automorphism $\sigma^{-1}$, i.e., for the skew power series ring $R^{\mathrm{op}}\left[\left[x ; \sigma^{-1}\right]\right]$. Since $R^{\mathrm{op}}\left[\left[x ; \sigma^{-1}\right]\right]$ is isomorphic to the opposite $\operatorname{ring} R[[x ; \sigma]]^{\text {op }}$ of the $\operatorname{ring} R[[x ; \sigma]]$, and the 
conditions (1)-(10) are "right-sided", it follows that $R[[x ; \sigma]]$ and $R$ satisfy the "left-sided" versions of (1)-(10), that is, the left analogues of (1)-(10).

To complete the proof it suffices to show that if $\sigma$ is bijective and for some $i \in\{1,2, \ldots, 10\}$ the left analogue of (i) is satisfied, then (12) is satisfied. But if the left analogue of (i) is satisfied, then from the isomorphism $R[[x ; \sigma]]^{\mathrm{op}} \simeq R^{\mathrm{op}}\left[\left[x ; \sigma^{-1}\right]\right]$ it follows that (i) is satisfied for $R^{\mathrm{op}}\left[\left[x ; \sigma^{-1}\right]\right], R^{\mathrm{op}}$ and $\sigma^{-1}$. Hence, by the first part of the theorem, (12) is satisfied for $R^{\mathrm{op}}$ and $\sigma^{-1}$. Thus (12) is also satisfied for $R$ and $\sigma$.

In the following corollary we continue the list of characterizations for the ring $R[[x ; \sigma]]$ to be right duo right Bézout, given in Theorem 1.6.

Corollary 3.1. Let $\sigma$ be an endomorphism of a ring $R$. Then the conditions (1) through (12) in Theorem 1.6 are equivalent to each of the following:

(13) $R[[x ; \sigma]]$ is right Bézout, $R$ is right duo and $\sigma$ is injective.

(14) $R[[x ; \sigma]]$ is right Bézout, $R$ is reduced and $\sigma$ is injective.

(15) $R[[x ; \sigma]]$ is right Bézout, $R$ is right quasi-duo and $\sigma$ is injective.

(16) $R[[x ; \sigma]]$ is right Bézout, $R$ is semicommutative and $\sigma$ is injective.

(17) $R[[x ; \sigma]]$ is right Bézout, $R$ is abelian and $\sigma$ is injective.

(18) $R[[x ; \sigma]]$ is right Bézout, $R$ is strongly regular and $\sigma$ is injective.

(19) $R$ is right duo right $\aleph_{0}$-injective von Neumann regular, $\sigma$ is bijective and $\sigma(e)=e$ for any $e=e^{2} \in R$.

Proof: As an immediate consequence of the implication chart $(*)$ given at the beginning of this section, we obtain (13) $\Rightarrow(15)$ and (14) $\Rightarrow$ (16) $\Rightarrow(17)$. Moreover, by $(*),(15)$ as well as (17) imply that $R$ is Dedekindfinite, and thus $R$ is von Neumann regular by Proposition 2.1. Since a von Neumann regular ring that is right quasi-duo or abelian must be strongly regular, it follows that (15) as well as (17) imply (18). Thus $(13) \Rightarrow(15) \Rightarrow(18)$ and $(14) \Rightarrow(16) \Rightarrow(17) \Rightarrow(18)$. On the other hand, it is clear that $(18) \Rightarrow(13)$ and $(18) \Rightarrow(14)$. This proves the equivalence of (13) through (18). Furthermore, from Theorem 1.4 it follows that (16) is equivalent to the condition (8) of Theorem 1.6.

Since strongly regular rings are precisely right duo von Neumann regular rings, it follows that (19) is equivalent to the condition (12) of Theorem 1.6. 
Remark 3.2. Let $\mathcal{C}$ be a class of rings such that for any $\operatorname{ring} R$ the following conditions are satisfied:

(a) If $R \in \mathcal{C}$, then $R$ is abelian or right quasi-duo.

(b) If $R$ is reduced, right duo and right Bézout, then $R \in \mathcal{C}$.

Then it easily follows from Theorem 1.6 and Corollary 3.1 that for any ring $R$ and an endomorphism $\sigma$ of $R$ the following conditions are equivalent to the equivalent conditions listed in Theorem 1.6 and Corollary 3.1:

(1) $R[[x ; \sigma]]$ is right Bézout, $R[[x ; \sigma]] \in \mathcal{C}$ and $\sigma$ is injective.

(2) $R[[x ; \sigma]]$ is right Bézout, $R \in \mathcal{C}$ and $\sigma$ is injective.

For instance, the conditions (a) and (b) are satisfied by the classes that appeared in Theorem 1.6, namely the classes of reduced rings, semicommutative rings, abelian rings, right duo rings, right quasi-duo rings and right distributive rings. Below we present three other classes satisfying (a) and (b):

- reversible rings, i.e. rings $R$ such that $a b=0 \Leftrightarrow b a=0$ for all $a, b \in R$ (see [2]);

- symmetric rings, i.e. rings $R$ such that $a b c=0 \Leftrightarrow a c b=0$ for all $a, b, c \in R$ (see [8]);

- Armendariz rings, i.e. rings $R$ such that whenever polynomials $f(x)=a_{0}+a_{1} x+\cdots+a_{m} x^{m}$ and $g(x)=b_{0}+b_{1} x+\cdots+b_{n} x^{n}$ in $R[x]$ satisfy $f(x) g(x)=0$, then $a_{i} b_{j}=0$ for all $i, j$ (see [11]).

The following example shows that in the last part of Theorem 1.6 the assumption that $\sigma$ is bijective is essential.

Example 3.3. Let $K=F\left(y_{1}, y_{2}, y_{3}, \ldots\right)$ be the rational function field in infinitely many variables $y_{n}$ over a field $F$, and let $\sigma$ be the endomorphism of $K$ defined by $\sigma\left(y_{n}\right)=y_{n+1}$ for any $n \in \mathbb{N}$, and $\sigma(f)=f$ for any $f \in F$. Then $\sigma$ is an injective endomorphism of $K$ that is not an automorphism. Set $A=K[[x ; \sigma]]$. If $g \in A \backslash\{0\}$, then $g$ can be written in the form $g=\left(k_{0}+k_{1} x+k_{2} x^{2}+\cdots\right) x^{n}$ with invertible $k_{0}$, and thus $A g=A x^{n}$. Now it easily follows that left ideals of $A$ are totally ordered by set inclusion, and thus $A$ is left distributive. Hence the left analogue of (8) is satisfied, whereas obviously (12) is not satisfied.

\section{Acknowledgments}

The authors thank two anonymous referees for helpful comments and suggestions for the improvement of this paper.

The first author was partially supported by the Flemish-Polish bilateral agreement BIL2005/VUB/06 and the Bialystok Technical University grant $\mathrm{W} / \mathrm{WI} / 3 / 06$. 


\section{References}

[1] J. W. Brewer, E. A. Rutter, And J. J. Watkins, Coherence and weak global dimension of $R[[X]]$ when $R$ is von Neumann regular, J. Algebra 46(1) (1977), 278-289.

[2] P. M. Cohn, Reversible rings, Bull. London Math. Soc. 31(6) (1999), 641-648.

[3] D. Herbera, Bezout and semihereditary power series rings, $J$. Algebra 270(1) (2003), 150-168.

[4] C. Y. Hong, N. K. Kim, And T. K. KwaK, Ore extensions of Baer and p.p.-rings, J. Pure Appl. Algebra 151(3) (2000), 215-226.

[5] O. A. S. Karamzadeh and A. A. KoochaKPoor, On $\aleph_{0}$-self-injectivity of strongly regular rings, Comm. Algebra 27(4) (1999), 1501-1513.

[6] J. Krempa, Some examples of reduced rings, Algebra Colloq. 3(4) (1996), 289-300.

[7] T. Y. LAM, "A first course in noncommutative rings", Graduate Texts in Mathematics 131, Springer-Verlag, New York, 1991.

[8] J. LAmBeK, On the representation of modules by sheaves of factor modules, Canad. Math. Bull. 14 (1971), 359-368.

[9] G. Marks, Duo rings and Ore extensions, J. Algebra 280(2) (2004), 463-471.

[10] R. Mazurek and M. Ziembowski, On Bezout and distributive generalized power series rings, J. Algebra 306(2) (2006), 397-411.

[11] M. B. Rege and S. Chhawchharia, Armendariz rings, Proc. Japan Acad. Ser. A Math. Sci. 73(1) (1997), 14-17.

[12] A. A. Tuganbaev, "Distributive modules and related topics", Algebra, Logic and Applications 12, Gordon and Breach Science Publishers, Amsterdam, 1999.

[13] A. A. Tuganbaev, Rings of series, and weak global dimension, (Russian), Izv. Vyssh. Uchebn. Zaved. Mat. 11 (1987), 70-78, 89; English translation: Soviet Math. (Iz. VUZ) 31(11) (1987), 90-101.

[14] A. A. Tuganbaev, Distributive rings and modules, (Russian), Mat. Zametki 47(2) (1990), 115-123, 160; translation in: Math. Notes 47(1-2) (1990), 199-206.

Ryszard Mazurek:

Faculty of Computer Science

Białystok Technical University

Wiejska 45A

15-351 Białystok

Poland

E-mail address: mazurek@pb.bialystok.pl 
Michał Ziembowski:

Current address:

Maxwell Institute of Sciences

School of Mathematics

University of Edinburgh

James Clerk Maxwell Building

King's Buildings, Mayfield Road

Edinburgh EH9 3JZ

Scotland, UK

E-mail address: m.ziembowski@sms.ed.ac.uk, m.ziembowski@wp.pl

Primera versió rebuda el 6 de febrer de 2008,

darrera versió rebuda el 27 d'octubre de 2008. 